\title{
Photo- and Electroreflectance Spectroscopy of Low-Dimensional III-Nitride Structures
}

\author{
A. DrabińSKA* \\ Institute of Experimental Physics, Warsaw University \\ Hoża 69, 00-681 Warsaw, Poland
}

\begin{abstract}
The review of electromodulation techniques such as photo- and electroreflectance spectroscopy is presented and illustrated using spectra of $\mathrm{AlGaN} / \mathrm{GaN}$ heterostructures. By using these techniques it is possible to analyze the Franz-Keldysh oscillations to obtain the value of electric field near the surface or at interface. When additionally a constant voltage is applied to the structure, it is possible to obtain the charge distribution or the gradient of polarization present in the sample, which is of the origin of $2 \mathrm{D}$ electron gas at the $\mathrm{AlGaN} / \mathrm{GaN}$ interface. The polarization is discussed assuming two contributions: spontaneous and piezoelectric polarization. Piezoelectric part of polarization can be obtained from X-ray diffraction, providing information about strain in the structure. Influence of light and voltage on $2 \mathrm{D}$ electron gas properties and depletion layer width is demonstrated using photocapacitance-voltage measurements. All results are discussed and compared with self-consistent calculations of potentials and electric fields in the structure.
\end{abstract}

PACS numbers: 77.22.Ej, 77.65.Ly, 78.40.Fy

\section{Introduction}

Modulation spectroscopy is the branch of optical spectroscopy that deals with the measurement and interpretation of changes in optical spectra caused by periodic modification of measurements condition. Modulation techniques can change some parameters of the sample (external modulation) or of the measuring system (internal modulation). A large number of techniques involving internal or

*e-mail: Aneta.Drabinska@fuw.edu.pl 
external modulations have been developed, in fact the limits of using the modulation depends on one's imagination. For example, modulation involves such parameters as wavelength, temperature, pressure, magnetic field, or electric field. In this work only one particular modulation will be presented i.e. electric field modulation, which, due to the acceleration mechanism, generates the third-derivative spectra and to illustrate this the spectra of $\mathrm{AlGaN} / \mathrm{GaN}$ heterostructures will be used. The basic rule that stands behind electromodulation reflectivity measurements is to modulate electric field present in a structure and to measure the change of reflectance during this process.

It has been predicted by theory [1-4] that group III-nitride semiconductors such as GaN and AlN in the wurtzite structure possess a very large spontaneous polarization. Although the spontaneous polarization is very strong, the pyroelectric coefficients, describing the change of spontaneous polarization with temperature are very small. This is considered to be a big advantage for using these materials for high power and high temperature devices. In case of III-nitride heterostructures in addition to spontaneous polarization the piezoelectric polarization is always present, and the piezoelectric constants of those materials are also very large. The effect of these two polarizations can have substantial influence on concentration and distribution of free carriers in strained heterostructures, for example for creation of two-dimensional electron gas (2DEG) on AlGaN/GaN interface. Observation of Franz-Keldysh oscillations (FKOs) in electroreflectance or photoreflectance spectra provides also direct evidence of the presence of electric fields caused by gradients in spontaneous and piezoelectric polarizations. The aim of this work was to review the electromodulation techniques such as photoand electroreflectance spectroscopy and to present possible to obtain information such as the magnitude of built-in electric field. In this paper, firstly modulation techniques are described and secondly samples used in this work and experimental results obtained from these techniques are presented.

\section{Experimental techniques}

The general concept of modulation spectroscopy is that an optical spectrum that contains many broad lines can be reduced to a series of derivative-like lines. In the case of electromodulation reflectivity (EMR) the measured parameter is a periodic variation in reflectivity caused by small periodic changes of electric field in the sample. The benefits of this technique is that it gives very sharp lines which are sensitive to surface and interfaces built-in electric field.

Considering the different manners of modulating the electric field, it is possible to distinguish several methods of measuring EMR. They can be divided into two categories: methods that require contacts on the sample [5] and contactless methods [6-9].

Electroreflectance (ER) technique requires at least two contacts on the structure and modulation voltage is applied between them so the internal electric field 
is modulated. There are at least two possible ways of modulating internal electric field. First, when both contacts are on the top of the structure and the electric field perpendicular to the direction of growth is modulated. This method called transverse electroreflectance (TER) can be applied only to nonconducting structures and requires very large modulating voltages. Another method of ER is connected with the Schottky barrier electroreflectance (SBER) or longitudinal electroreflectance, when one of the contacts is made on the back side of a substrate and another contact is a semitransparent Schottky contact on the top of the structure (Fig. 1a). In this case the electric field parallel to the growth direction is modulated and ER is measured in the depleted region. In this method in addition to the applied modulation voltage it is also possible to apply constant voltage. That produces external field in the sample and changes the depletion layer width.
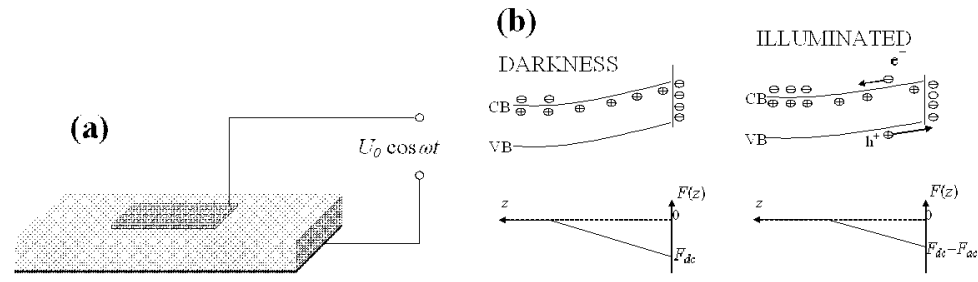

Fig. 1. The scheme of the contacts for modulation of electric field in the Schottky barrier electroreflectance (a) and the principle of the modulation of electric field using the illumination with chopped laser in photoreflectance (b).

Another EMR method is contactless electroreflectance (CER) where the sample is placed in capacitor with one semitransparent electrode biased with modulating voltage. However, due to the big distance between electrodes it requires large modulating voltages.

Photoreflectance (PR) is a contactless form of electromodulation reflectivity; the electric field is modulated by illuminating the sample periodically with monochromatic light of energy above the band gap. PR uses the following phenomenon. In thin layer illumination with light of energy larger than the band gap of material, produces the pairs of free carriers which due to internal electric field are separated, and thereby reduce that field and flatten the bands (Fig. 1b).

It is also possible to measure PR under the Schottky barrier biased with a constant voltage. In this case, the Schottky barrier photoreflectance (SBPR) is measured on the structure in external electric field.

Although the modulation of electric field can be performed in several ways the method of measuring EMR is the same in every case (Fig. 2). The reflectivity is measured with a monochromatic light obtained in this case from a xenon lamp. The light of intensity $I_{0}$ is focused on the modulated sample and the reflected light is detected by silicon detector. The intensity signal consists of dc value $I_{0} R(E)$ and ac value $I_{0} \Delta R(E)$, measured by a voltmeter and lock-in amplifier synchronized 
with the modulation source, respectively. Dividing both the signals eliminates the uninteresting common factor $I_{0}$, and then yields the quantity $\Delta R / R(E)$. In this case the PR measurements are performed with modulating sample with a chopped He-Cd laser. In the case of SBER and SBPR measurements the Schottky barrier was obtained by evaporating a thin semitransparent gold contact on top of the structure and alloyed indium ohmic contact made connection to all conductive layers inside the structure. Between these two contacts the modulating voltage was applied. SBER and SBPR measurements were performed also as a function of constant voltages applied to the Schottky contact.

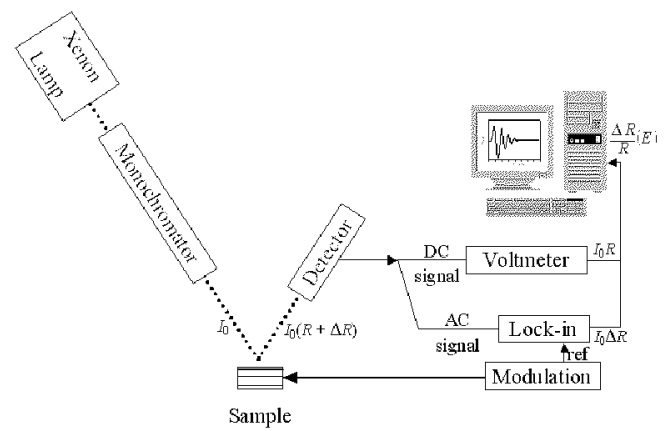

Fig. 2. The scheme of experimental setup used for the electromodulation reflectance.

Schottky barrier electroreflectance has been measured using the electroreflectance bias-wavelength (ERBW) mapping technique [10] that has been already successfully applied to nitride structures [11-13]. The clue of this method is that ER signal is measured first at given wavelength for different constant voltages applied to the Schottky contact. After this the wavelength is changed and again the $\mathrm{ER}$ is measured for different voltages.

\section{Theoretical considerations of electromodulation spectroscopy}

A change of the electric field induces the perturbation of the dielectric function.

The differential changes of the reflectivity are related with the dielectric function by equation [14]:

$$
\frac{\Delta R}{R}=\alpha \Delta \varepsilon_{1}+\beta \Delta \varepsilon_{2},
$$

where $\alpha$ and $\beta$ are the Seraphin coefficients correlated with unperturbed dielectric function $\left(\varepsilon=\varepsilon_{1}+\mathrm{i} \varepsilon_{2}\right)$, and $\Delta \varepsilon_{1}+\mathrm{i} \Delta \varepsilon_{2}$ is perturbation induced change of the dielectric function. In general, $\alpha$ and $\beta$ are nonzero quantities but near the fundamental gap of bulk materials $\beta \approx 0[15,6]$. However, the Seraphin coefficients are sensitive to optical interference effects, so in multilayer structures they are 
modified and $\beta$ is not negligible. The exact form of $\Delta \varepsilon_{1}$ and $\Delta \varepsilon_{2}$ can be calculated if the dielectric function and type of the critical point in the band structure is known.

Electromodulation can be classified into three categories depending on the relative strengths of characteristic energies of the unperturbed system and of the system in the electric field $(F)$ for intra-band and inter-band processes. In inter-band processes the characteristic energy of the unperturbed system is the value of energy gap $\left(E_{\mathrm{g}}\right)$, and the characteristic energy of system in the electric field is a drop of potential $\left(V^{F}\right)$ in elementary cell caused by electric field

$$
V^{F}=e F a_{0}
$$

where $a_{0}$ is the lattice constant.

For intra-band processes the characteristic energy of the system is the broadening parameter $(\Gamma)$ connected with the average lifetime $(\tau)$ of the carriers on given energy level, and

$$
\Gamma \tau=\hbar \text {. }
$$

For the system in electric field - the electro-optic energy $(\hbar \Omega)$ is given by

$$
(\hbar \Omega)^{3}=\frac{e^{2} \hbar^{2} F^{2}}{8 \mu_{\|}}
$$

and the physical interpretation of this energy is the energy of accelerated particle with mass $\mu_{\|}$parallel to the field direction in uniform electric field.

In low-field limit both field characteristic energies are much smaller than the system characteristic energies ( $\hbar \Omega \ll \Gamma$ and $V^{F} \ll E_{\mathrm{g}}$ ). In this case very characteristic third-derivative line shapes appear. If characteristic energy of the field is smaller than the one corresponding to the system for the inter-band processes, but larger for intra-band processes $\left(\hbar \Omega \geq \Gamma\right.$ and $V^{F} \ll E_{\mathrm{g}}$ ), this is the limit of intermediate field. In this case it is possible to observe the Franz-Keldysh oscillations. In high-field limit both field characteristic energies are comparable or larger than the characteristic energies of the system $\left(\hbar \Omega \geq \Gamma\right.$ and $\left.V^{\mathrm{F}} \geq E_{\mathrm{g}}\right)$, in this case the Stark effect appears.

In heterostructures it is possible to obtain few signals originating from different regions in the structure. In this case it is very difficult to discuss that kind of spectrum, especially if they are at the same energy, so there were suggested methods allowing to separate these signals $[16,17]$.

\subsection{Low-field limit}

Considering the full form of dielectric function $\varepsilon(E, \hbar \Omega, \Gamma)$ the changes in dielectric function caused by the perturbation of electric field can be expressed by $[18]$ :

$$
\Delta \varepsilon \approx \frac{(\hbar \Omega)^{3}}{3 E^{2}} \frac{\partial^{3}}{\partial E^{3}}\left[E^{2} \varepsilon\left(E-E_{\mathrm{g}}, \Gamma\right)\right] .
$$


This form of $\Delta \varepsilon$ explains not only the anomalous sharpness of photoreflectance and electroreflectance spectra, but also shows that in this case the line shape of $\Delta \varepsilon$ is independent of electric field. Its amplitude increases as the square of electric field and is inversely proportional to the interband reduced effective mass in electric field direction.

For the case of homogeneous broadening parameter the dielectric function has a generalized Lorentzian form. For this case the photoreflectance and electroreflectance signal is expressed by the following formula:

$$
\frac{\Delta R}{R}=(\hbar \Omega)^{3} \operatorname{Re}\left[A \mathrm{e}^{\mathrm{i} \varphi}\left(E-E_{\mathrm{g}}+\mathrm{i} \Gamma\right)^{-m}\right],
$$

where $A$ and $\varphi$ are the amplitude and phase factor, respectively, and the power $m$ depends on the type of a critical point (for example for a three-dimensional critical point $m=2.5$ while for a two-dimensional critical point $m=3$ ).

In practice the low-field limit is realized if $|\hbar \Omega|^{3} / \Gamma^{3}<1 / 3$ or $|\Delta R / R|<10^{-3}$.

\subsection{Intermediate-field limit}

For the case when electro-optic energy is no longer smaller than the broadening parameter the expression describing the line shape of electroreflectance and photoreflectance spectra $[6,19]$ is given by the following formula:

$$
\frac{\Delta R}{R} \propto \frac{1}{E^{2}\left(E-E_{\mathrm{g}}\right)} \exp \left[-\Gamma \sqrt{\frac{E-E_{\mathrm{g}}}{(\hbar \Omega)^{3}}}\right] \cos \left[\frac{2}{3} \sqrt{\left(\frac{E-E_{\mathrm{g}}}{\hbar \Omega}\right)^{3}}+\varphi\right] .
$$

This formula describes oscillations observed in ER and PR spectra which are called the Franz-Keldysh oscillations. The period of these oscillations is proportional to the electric field and inversely proportional to the square root of reduced effective mass in the electric field direction. The amplitude of these oscillations vanishes exponentially and depends not only on the electric field but also on the broadening parameter.

The linear dependence of $\left(E-E_{\mathrm{g}}\right)_{n}^{3 / 2}$ as a function of the index number $n$ of the FKOs extremes allows determination of the value of the electro-optic energy. This is equivalent to determination of relation between value of the electric field and reduced effective mass in the field direction. Analysis of the FKOs amplitude gives further information about the broadening parameter.

In the presence of large dc electric field with small modulation component ( $F_{\text {ac }} \ll F_{\text {dc }}$ ) the period of FKOs is determined by $F_{\text {dc }}$. On the other hand, in the case of a very small dc electric field $\left(F_{\mathrm{dc}} \ll F_{\mathrm{ac}}\right)$, the FKOs period is determined by the modulating field $F_{\text {ac }}$. However, if modulation of electric field is not small comparing to the dc electric field, first few FKOs are still determined by $F_{\text {dc }}[20,21]$. 


\subsection{Bound states}

For carriers in bound states, like quantum wells, the effective mass along the field direction is infinitive and electro-optic energy vanishes. These types of particles are confined in space and the electric field is not able to accelerate them. In this case the effect of an electric field applied along direction of confinement is much different than it was described previously. For example, considering the square quantum well, the effect of the electric field along the confinement direction adds a linear potential, which tilts the quantum well and the barriers, thus changing the shape of the potential well. The wave functions are mixed in such a way that electrons and holes are shifted in opposite directions, but they are still confined; in addition, energy of their states is changed as well. In high electric field the quantum well may be perturbed in a way that tunneling may take place.

For bound states the changes in the dielectric function caused by modulation of electric field may be expressed as [22, 23]:

$$
\Delta \varepsilon=\left[\frac{\partial \varepsilon}{\partial E_{\mathrm{g}}} \frac{\partial E_{\mathrm{g}}}{\partial F_{\mathrm{ac}}}+\frac{\partial \varepsilon}{\partial \Gamma} \frac{\partial \Gamma}{\partial F_{\mathrm{ac}}}+\frac{\partial \varepsilon}{\partial I} \frac{\partial I}{\partial F_{\mathrm{ac}}}\right] F_{\mathrm{ac}},
$$

where $I$ is the intensity of the optical transition.

In this case, the measured ER or PR signal is a function of six parameters, and is connected with first-derivative spectrum. Its amplitude is proportional to the modulating electric field $\left(F_{\mathrm{ac}}\right)$. This - the most general form of the modulated dielectric function for confined systems - includes the possibility of modulation of lifetime by electric field, caused for example by tunneling.

\section{Experimental results and discussion}

\subsection{Structure}

The investigated Ga-face AlGaN/GaN heterostructures have been grown by metal-organic chemical vapor deposition (MOCVD) on a sapphire substrate. The scheme of the structure is presented in Fig. 3. Intentionally undoped $\mathrm{Al}_{0.11} \mathrm{Ga}_{0.89} \mathrm{~N} / \mathrm{GaN}$ heterostructures were grown on thick $\mathrm{Si}$ doped GaN layer. The nominal donor concentration of GaN:Si layer was $N_{\mathrm{D}}=2.0 \times 10^{18} \mathrm{~cm}^{-3}$.

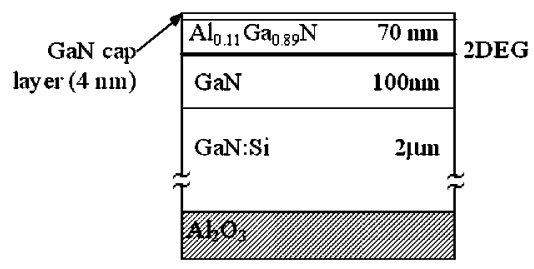

Fig. 3. The scheme of the AlGaN/GaN heterostructure grown on the thick conductive Ga.N layer. 
A very thin GaN cap layer was grown on the top of the structure to protect AlGaN layer.

Thermally evaporated semitransparent gold Schottky contact was placed on the structure surface. Alloyed ohmic contacts made connection to the doped GaN layer and to 2DEG on AlGaN/GaN interface as well.

All measurements were performed at room temperature.

\subsection{Photoreflectance and electroreflectance spectra}

The following EMR measurements: photoreflectance, Schottky barrier electroreflectance, and Schottky barrier photoreflectance, have been performed on the investigated structure. Results of the SBER were already presented before [13], however, for clarity they will be also discussed here. Figure 4 presents the PR spectrum of the investigated structure. The peak at energy $3.419 \mathrm{eV}$ is related to transitions in the undoped GaN layer, and the peak at energy $3.764 \mathrm{eV}$ is related to transitions in the undoped AIGaN layer. Both of these peaks show characteristic, of the low-field limit, line shape. However, in the examined spectrum the third feature is clearly visible at energy $3.458 \mathrm{eV}$. This feature was identified previously [24] as originating from the 2DEG on AlGaN/GaN interface. The clear evidence of the origin of this peak can be seen in Fig. 5. In this figure the photoreflectance is presented for the energy range containing GaN and the 2DEG related peak for different reverse bias applied to the Schottky diode. For low reverse bias both

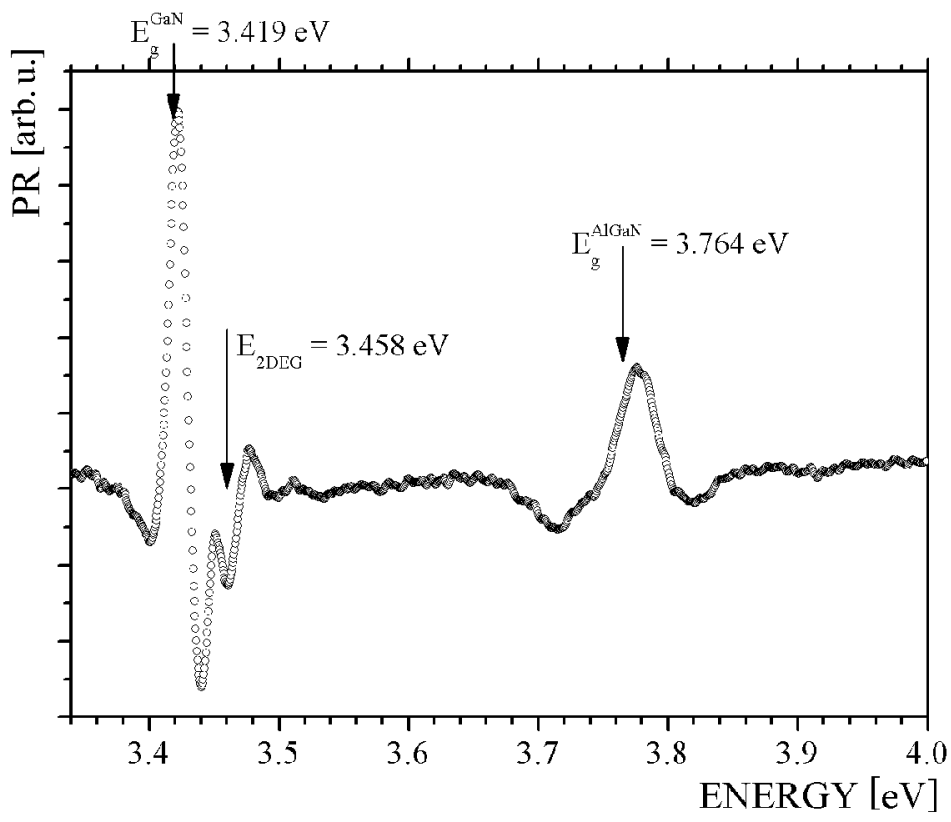

Fig. 4. Photoreflectance spectrum of AlGaN/GaN heterostructure. 


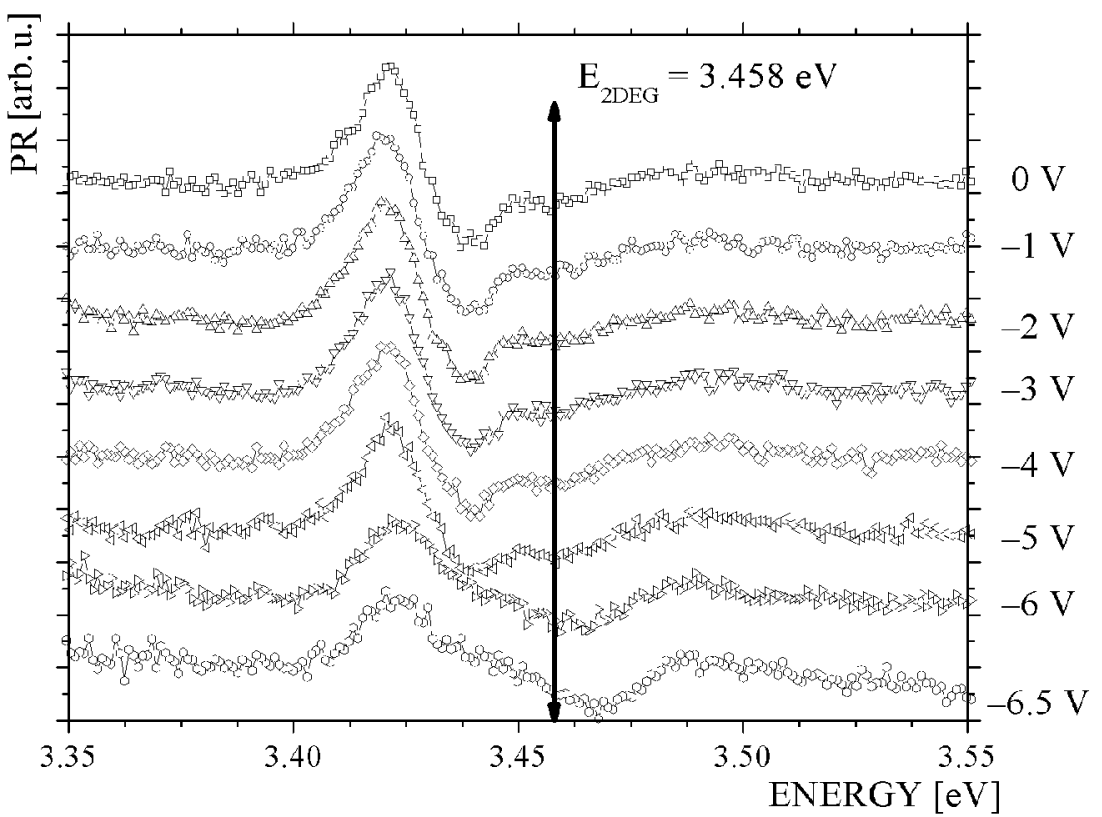

Fig. 5. Schottky barrier photoreflectance of $\mathrm{AlGaN} / \mathrm{GaN}$ heterostructure for different polarizations of the Schottky barrier.

peaks are visible. With applied higher reverse voltage, due to depletion of 2DEG from AlGaN/GaN interface the 2DEG related peak vanishes, and the GaN related peak is changing its shape to characteristic Franz-Keldysh oscillations. The photoreflectance spectra can be explained in the following way: in the absence of the Schottky barrier there is no electric field present in the structure. For low reverse bias, due to the presence of $2 \mathrm{DEG}$, the electric field generated by the surface barrier and the applied bias is present only in AlGaN layer. For high bias the 2DEG is removed from the interface, and the electric field in GaN layer becomes present. This is the cause of the change in line shape of the GaN related peak. The characteristic voltage at which $2 \mathrm{DEG}$ is depleted is over $-5 \mathrm{~V}$.

The above interpretation agrees with the SBER spectra presented in Fig. 6a as the ERBW map and in Fig. 6b as the cross-section of ERBW map for chosen biases. For low reverse bias one set of FKOs is clearly visible at energy over $3.7 \mathrm{eV}$. These are oscillations from AlGaN layer, and they are visible for all reverse bias applied to the Schottky contact. An increase in their period is clearly seen, which can be translated as an increase in electric field in the AlGaN layer. For higher reverse bias another peak emerges at energy about $3.42 \mathrm{eV}$ and has a shape characteristic of the low-field regime. This is the peak from the undoped GaN layer. Again this could be understood as an effect of depletion of 2DEG from the $\mathrm{AlGaN} / \mathrm{GaN}$ interface at some critical reverse voltage, so the electric field can now penetrate GaN layer. 

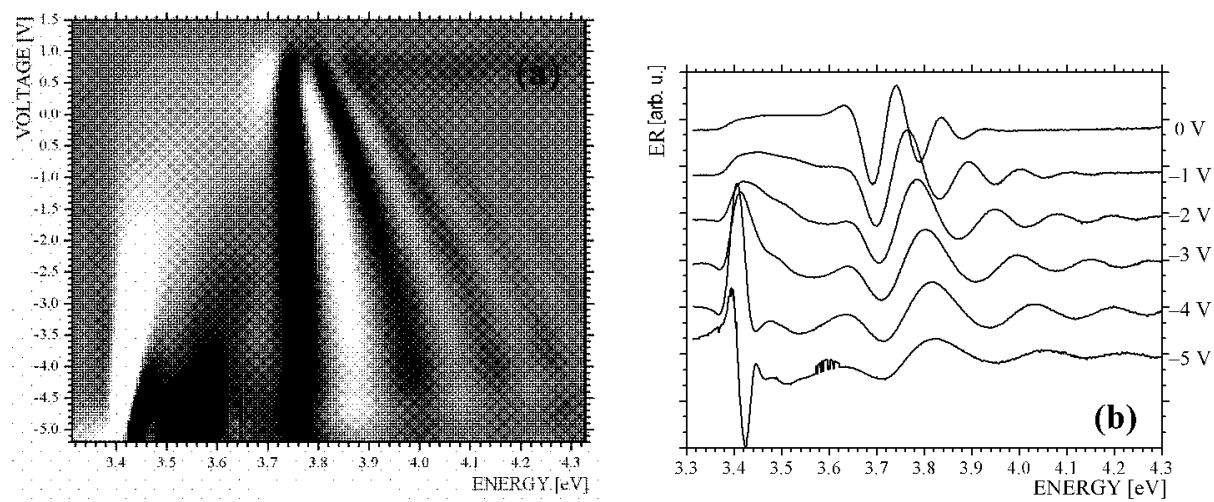

Fig. 6. (a) Schottky barrier electroreflectance map of $\mathrm{AlGaN} / \mathrm{GaN}$ heterostructures. Brighter (darker) areas represent the positive (negative) values of SBER signal. (b) The cross-sections of SBER map for different polarizations of the Schottky barrier.

The Fourier transform analysis of SBER spectra (with the value of reduced effective mass in field direction equal to $0.18 m_{0}$ [25]) allows obtaining the electric field dependence on the bias in the $\mathrm{AlGaN}$ layer. The results are presented in Fig. 7. For the low reverse bias the electric field increases linearly from about 140 to about $650 \mathrm{kV} / \mathrm{cm}$.

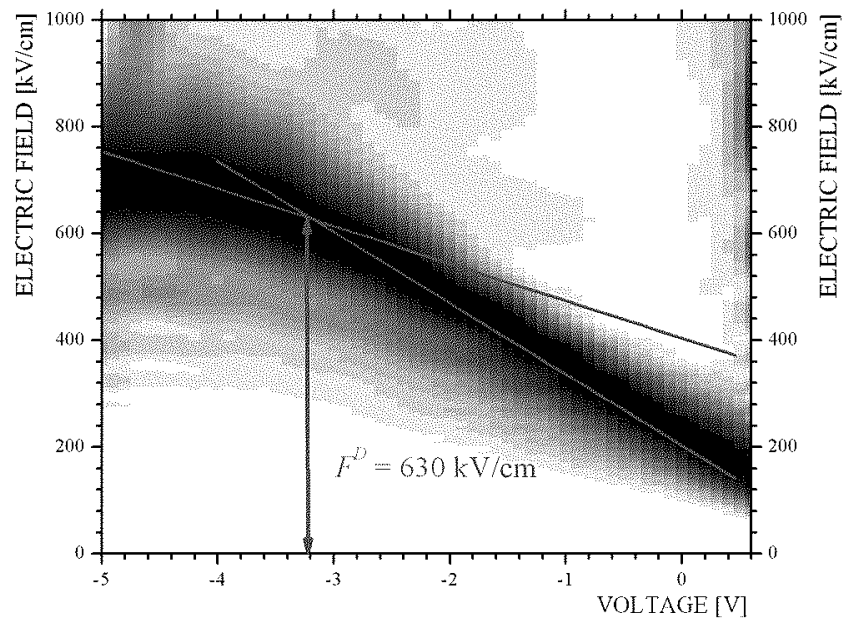

Fig. 7. The calculated Fourier transform from the FKOs in AlGaN layer. The dark areas represent maximum of the Fourier transform spectrum and indicate the value of electric field in AlGaN layer. The linear fits of electric field assuming constant width of depletion layer are plotted as solid lines. 
The linear dependence on bias can be explained assuming constant width of depleted layer $d$. The best fit to the formula

$$
F=\frac{-U+U_{\mathrm{B}}}{d}
$$

gives the depleted layer width $d_{1}=(75 \pm 5) \mathrm{nm}$ and the Schottky barrier height $U_{\mathbf{B}}=(1.5 \pm 0.2) \mathrm{V}$. The depletion width is in very good agreement with the nominal position of $\mathrm{AlGaN} / \mathrm{GaN}$ interface. A large electric field effectively removes all electrons from the AlGaN/GaN interface. For bias higher that $-3 \mathrm{~V}$ the slope decreases, and the electric field increases to about $800 \mathrm{kV} / \mathrm{cm}$ for bias $-5 \mathrm{~V}$. The fitted depletion width now obtained is $d_{2}=(145 \pm 20) \mathrm{nm}$, which is the position of thick Si doped GaN layer. Therefore, at the critical voltage over $-3 \mathrm{~V}$, 2DEG is removed from interface by electric field induced by voltage applied to the Schottky contact. The electric field $\left(F^{\mathrm{D}}\right)$ at which it happens is connected with the difference of polarization between AlGaN and GaN layers, that equal to $\Delta P^{\mathrm{Exp}}=0.0053 \mathrm{C} / \mathrm{m}^{2}$ and the concentration of the polarization positive electric charge located on the interface [25] equal to $\rho^{+}=3.3 \times 10^{12} \mathrm{~cm}^{-2}$.

Polarization of AlGaN and GaN consists of two components: spontaneous and piezoelectric polarization.

The X-ray diffraction was performed to obtain the lattice parameters which allow to calculate the piezoelectric polarization caused by the strain in the structure. The piezoelectric polarization can be calculated by using the piezoelectric coefficients $\left(e_{33}, e_{31}\right)[26]$ and equilibrium lattice parameters $\left(c_{0}, a_{0}\right)[27]$ as

$$
P^{\mathrm{PE}}=e_{33} \frac{c-c_{0}}{c_{0}}+2 e_{31} \frac{a-a_{0}}{a_{0}},
$$

where $c$ and $a$ are the measured values of the lattice parameters. All lattice parameters and calculated piezoelectric polarizations are presented in Table.

TABLE

Equilibrium lattice parameters $\left(c_{0}, a_{0}\right)$, piezoelectric constants $\left(e_{31}, e_{33}\right)$, measured lattice parameters $(c, a)$ and calculated piezoelectric polarization $\left(P^{\mathrm{PE}}\right)$ of $\mathrm{GaN}$ and AlGaN. Equilibrium lattice parameters and piezoelectric constants of AlGaN were calculated using Vegard's rule.

\begin{tabular}{l|c|c|c|c|c|c|c}
\hline \hline & $c_{0}[\AA]$ & $a_{0}[\AA]$ & $e_{31}\left[\mathrm{C} / \mathrm{m}^{2}\right]$ & $e_{33}\left[\mathrm{C} / \mathrm{m}^{2}\right]$ & $c[\AA]$ & $a[\AA]$ & $P^{\mathrm{PE}}\left[\mathrm{C} / \mathrm{m}^{2}\right]$ \\
\hline $\mathrm{GaN}$ & 5.2262 & 3.1986 & -0.34 & 0.67 & 5.1947 & 3.1723 & 0.0015 \\
$\mathrm{AlN}$ & 4.9939 & 3.1095 & -0.53 & 1.50 & - & - & - \\
$\mathrm{AlGaN}$ & 5.2006 & 3.1888 & -0.36 & 0.76 & 5.1631 & 3.1723 & -0.0018
\end{tabular}

The difference of spontaneous polarization between AlGaN and GaN layers

$$
\Delta P^{\mathrm{SP}}=P_{\mathrm{GaN}}^{\mathrm{SP}}-P_{\mathrm{AlGaN}}^{\mathrm{SP}}=\Delta P^{\mathrm{Exp}}-\left(P_{\mathrm{GaN}}^{\mathrm{PE}}-P_{\mathrm{AlGaN}}^{\mathrm{PE}}\right)
$$

equals to $\Delta P^{\mathrm{SP}}=0.0020 \mathrm{C} / \mathrm{m}^{2}$. 
Theoretical calculations [3, 28] give the value of spontaneous polarization in $\mathrm{GaN}$ equal to $-0.034 \mathrm{C} / \mathrm{m}^{2}$, and in AlN $-0.090 \mathrm{C} / \mathrm{m}^{2}$. Although the Vegard law is valid in predicting the macroscopic structural properties of alloys like lattice constants, the electronic properties such as band gap and polarization are nonlinear functions of the alloy composition. The theoretical calculations, to second order in $x$ give for AlGaN layers the value of spontaneous polarization by expression

$$
P_{\mathrm{AlGaN}}^{\mathrm{SP}}=-0.090 x-0.034(1-x)+0.021 x(1-x) .
$$

Higher-order terms are neglected and their effect was estimated to be less than $10 \%$.

For the AlGaN layer with $11 \%$ contents of $\mathrm{Al}$, calculated value of spontaneous polarization is equal to $0.038 \mathrm{C} / \mathrm{m}^{2}$. Therefore the difference of spontaneous polarization between $\mathrm{GaN}$ and $\mathrm{AlGaN}$ predicted by theory is equal to $0.004 \mathrm{C} / \mathrm{m}^{2}$.

This value is in agreement with the value obtained from SBER measurements.

\subsection{The effect of illumination and voltage on depletion layer and carrier localization}

To show the effect of illumination on the structure properties, such as the depletion layer width, photocapacitance-voltage (PCV) characteristics were performed under illumination of the Schottky barrier to simulate the PR and ER measurements. From this characteristic the depletion layer width was calculated as a function of illumination energy and voltage applied to the Schottky contact $d(E, V)$. In Fig. 8a there is presented the calculated depletion layer width-voltage characteristics in four characteristic cases: (i) in the darkness, (ii) after illumination of the Schottky contact with monochromatic light with energy just above the GaN band gap, (iii) after illumination of the Schottky contact with monochromatic light with energy just above the AlGaN band gap and (iv) after illumination with UV laser. In the darkness, for low reverse bias, the depletion layer width agrees with the position of $\mathrm{AlGaN} / \mathrm{GaN}$ interface, so the $2 \mathrm{DEG}$ is present on the interface and the depletion layer is extended only to AlGaN layer. By applying higher voltage the depletion layer width increases - the $2 \mathrm{DEG}$ is depleted from the interface and the depletion layer extends into GaN layer, reaching finally thick Si doped GaN layer. Figure $8 \mathrm{~b}$ presents the depletion layer width as a function of monochromatic illumination of the Schottky contact, as it was in ER measurements for two voltage biases 0 and $-4 \mathrm{~V}$. For zero reverse bias, the depletion layer width is constant regardless of illumination and agrees with the position of the AlGaN/GaN interface. For high reverse voltage bias the illumination affects the depletion layer width. First, illuminating the Schottky contact with light of energy above the GaN band gap the depletion layer width decreases. After illuminating the Schottky barrier with light of energy above the AlGaN band gap the depletion layer width decreases even more. The depletion layer width as a function of voltage for these two characteristic light energies is presented in Fig. 8a. For illumination above GaN band gap the characteristic voltage at which 2DEG is depleted 

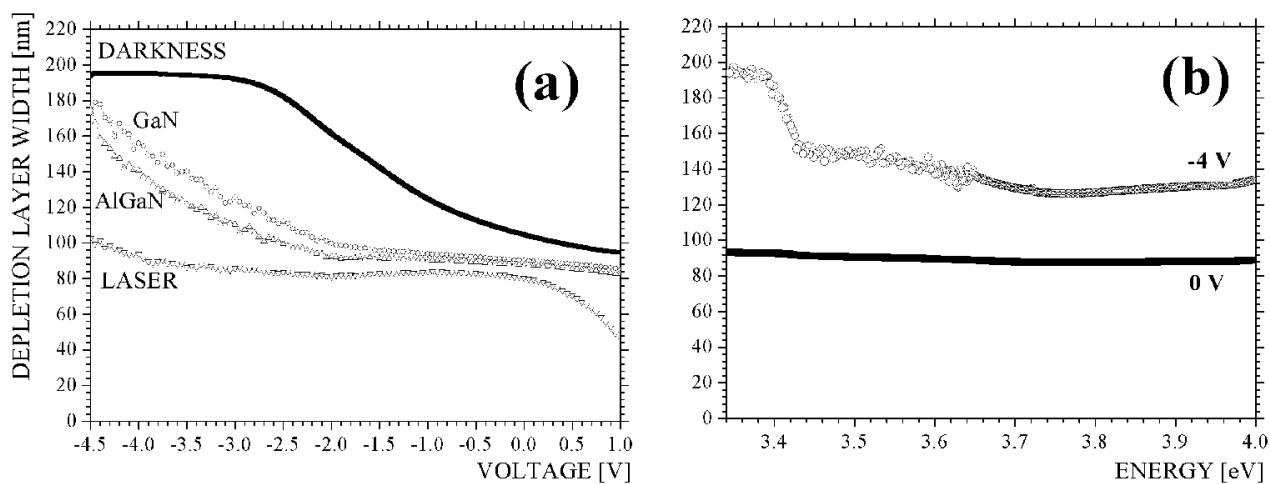

Fig. 8. Depletion layer width calculated from photocapacitance-voltage measurement in darkness, and under illumination with light of above the band gap of GaN and AlGaN and with UV laser (a). The dependence of the depletion layer width on energy of light for two biases: 0 and $-4 \mathrm{~V}$ (b).

is shifted by $2 \mathrm{~V}$ into higher reverse bias. It is even more shifted (by $2.5 \mathrm{~V}$ ) for illuminating above AlGaN band gap. The illumination of the Schottky contact with laser shifts the characteristic reverse voltage about $4.5 \mathrm{~V}$ comparing to the darkness. Therefore the monochromatic light with energy above the band gap of GaN or AlGaN produces additional electrons that are attracted to the AlGaN interface and hence more applied voltage is required to deplete electrons from the interface. These results are qualitatively consistent with previous results of the SBER and SBPR.

\subsection{Self-consistent calculations}

The experimental results can be compared with theoretical calculations based on the simple numerical model. Electric charge is a source of electric field that changes the potential along the structure, on the other hand, the concentration of the charged particles depends upon potential, so the charge, electric field, and the potential distributions must be calculated simultaneously by a self-consistent procedure.

The calculations were performed to simulate the capacitance-voltage characteristics in the darkness and calculated parameters were compared with the ones obtained from EMR measurements.

Potential was calculated as a space integral of the electric field

$$
V(z)=\int_{0}^{z} e F\left(z^{\prime}\right) \mathrm{d} z^{\prime}
$$

Using this potential, the concentration of electrons and holes and the occupancy of donors and acceptors given by the Fermi-Dirac distribution and the gradient of polarization were calculated. The electric field generated by the bias 

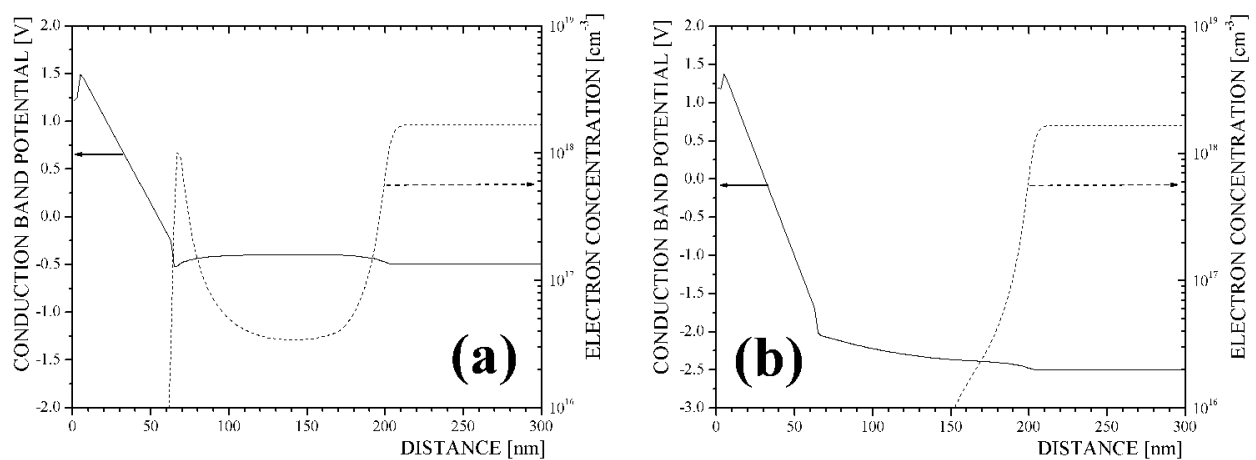

Fig. 9. Calculated conduction band potential (solid lines) and electron concentration (dashed lines) distributions for low reverse bias $-0.5 \mathrm{~V}$ (a) and for high reverse bias $-2.5 \mathrm{~V}$, (b) under assumption of no illumination.

applied to the sample and by electrical charges in the structure was calculated from the following formula:

$$
F(z)=\frac{U}{d}+\frac{1}{\varepsilon_{0} \varepsilon_{\mathrm{r}}} \int_{0}^{z} \rho\left(z^{\prime}\right) \mathrm{d} z^{\prime} .
$$

The calculated potential and electron concentration for the low reverse bias applied to the Schottky contact are presented in Fig. 9a. For this case, just below the $\mathrm{AlGaN} / \mathrm{GaN}$ interface there is formed a V-shaped potential well in which 2DEG is formed. The electrons screen the electric field, so there is no field in GaN layer. When a sufficiently high reverse bias is applied (Fig. 9b), the V-shaped potential well vanishes, electrons are removed, and the electric field penetrates into GaN layer.

\section{Conclusions}

The electromodulation techniques such as electroreflectance and photoreflectance were presented and discussed. It was shown that photoreflectance provides information about screening of the built-in electric field and allows determining the presence of $2 \mathrm{DEG}$ on AlGaN/GaN interface. In addition, the Schottky barrier electroreflectance and photoreflectance demonstrate depletion of 2DEG from $\mathrm{AlGaN} / \mathrm{GaN}$ interface during polarization of the Schottky barrier. However, the photoreflectance measurements are affected by creation, by the intense light, of additional electrons that are attracted to the interface. Even monochromatic light in electroreflectance creates the additional electrons in the structure. Electroreflectance (SBER) measurement and the Fourier transform analysis of FKOs in $\mathrm{AlGaN}$ layer allowed to obtain the electric field dependence on the bias in AlGaN, and therefore, allowed to determine the Schottky barrier height and the difference of polarization between $\mathrm{AlGaN}$ and GaN layers. Combining X-ray diffraction, which gave the strain and piezoelectric polarization of those layers, with 
SBER measurements allowed to calculate the difference of spontaneous polarization between AlGaN and GaN. The consistence of electromodulation reflectance measurements, the photocapacitance-voltage characteristics, and numerical calculations indicate how reliable are electromodulation reflectance techniques for investigation of low-dimensional semiconductor structures.

\section{References}

[1] F. Bernardini, V. Fiorentini, D. Vanderbilt, Phys. Rev. B 56, R10024 (1997).

[2] F. Bernardini, V. Fiorentini, Phys. Rev. B 64, 085207-1 (2001).

[3] O. Ambacher, J.A. Majewski, C. Miskys, A. Link, M. Hermann, M. Eickhoff, M. Stutzmann, F. Bernardini, V. Fiorentini, V. Tilak, B. Schaff, L.F. Eastman, J. Phys., Condens. Matter 14, 3399 (2002).

[4] O. Ambacher, J. Phys. D, Appl. Phys. 31, 2653 (1998).

[5] D.E. Aspnes, A.A. Studna, Phys. Rev. B 7, 4605 (1973).

[6] M. Cardona, K.L. Shaklee, F.H. Pollak, Phys. Rev. 154, 696 (1967).

[7] J.G. Gay, L.T. Klauder, Phys. Rev. 172, 811 (1968).

[8] X. Yin, F.H. Pollak, Appl. Phys. Lett. 59, 2305 (1991).

[9] J. Misiewicz, P. Sitarek, G. Sek, Introduction to the Photoreflectance Spectroscopy of Semiconductor Structures, Oficyna Wydawnicza Politechniki Wrocławskiej, Wrocław 1999.

[10] T. Tomaszewicz, A. Babiński, D. Suska, J.M. Baranowski, A. Tomaszewicz, Appl. Phys. Lett. 75, 2088 (1999).

[11] A. Drabińska, K.P. Korona, R. Bożek, A. Babiński, J.M. Baranowski, W. Pacuski, R. Stępniewski, T. Tomaszewicz, Phys. Status Solidi B 234, 868 (2002).

[12] A. Drabińska, A. Babiński, T. Tomaszewicz, R. Bożek, J.M. Baranowski, J. Appl. Phys. 92, 163 (2002).

[13] A. Drabińska, K.P. Korona, R. Bożek, J.M. Baranowski, K. Pakuła, T. Tomaszewicz, J. Gronkowski, Phys. Status Solidi C 0, 329 (2002).

[14] B.O. Seraphin, N. Bottka, Phys. Rev. 145, 628 (1996).

[15] D.E. Aspnes, Surf. Sci. 37, 418 (1973).

[16] S.L. Tyan, Y.C. Wang, J.S. Hwang, Appl. Phys. Lett. 68, 3452 (1996).

[17] K. Jezierski, J. Misiewicz, P. Markiewicz, M. Panek, B. Ściana, M. Tłaczała, R. Korbutowicz, Phys. Status Solidi A 147, 467 (1995).

[18] D.E. Aspnes, in: Handbook on Semiconductors, Vol. 2, Ed. M. Balkanski, North-Holland, Amsterdam 1980, Ch. 4A, p. 109.

[19] D.E. Aspnes, Phys. Rev. B 10, 4228 (1974).

[20] R.N. Bhattacharya, H. Shen, P. Parayanthal, F.H. Pollak, T. Coutta, H. Aharoni, Phys. Rev. B 37, 4044 (1988).

[21] H. Shen, F.H. Pollak, Phys. Rev. B 42, 7097 (1990).

[22] B.V. Shanabrook, O.J. Glembocki, W.T. Beard, Phys. Rev. B 35-I, 2540 (1987).

[23] R. Enderlein, D.S. Jiang, Y.S. Tang, Phys. Status Solidi B 145, 167 (1988). 
[24] A. Wójcik, T. Piwonski, T.J. Ochalski, M. Bugajski, A. Grzegorczyk, L. Macht, S. Haffoutz, P.K. Larsen, Phys. Status Solidi C 0, 491 (2002).

[25] J.A. Majewski, G. Zandler, P. Vogl, Acta Phys. Pol. A 100, 249 (2001).

[26] A.F. Wright, J. Appl. Phys. 82, 2833 (1997).

[27] A. Zoroddu, F. Bernardini, P. Ruggerone, V. Fiorentini, Phys. Rev. B 64, 045208-1 (2001).

[28] V. Fiorentini, F. Bernardini, O. Ambacher, Appl. Phys. Lett. 80, 1204 (2002). 